OPEN ACCESS

Edited by:

Yaxiong Zhang,

Sun Yat-sen University Cancer Center

(SYSUCC), China

Reviewed by:

Matteo Giaj Levra,

Centre Hospitalier Universitaire de

Grenoble, France

Santiago Viteri,

Instituto Oncológico Dr Rosell, Spain

${ }^{*}$ Correspondence:

Sabine Zöchbauer-Müller sabine.zoechbauer-mueller@ meduniwien.ac.at

Specialty section:

This article was submitted to

Thoracic Oncology,

a section of the journal

Frontiers in Oncology

Received: 11 August 2020 Accepted: 16 November 2020

Published: 26 January 2021

Citation:

Zöchbauer-Müller S, Kaserer B, Prosch H, Cseh A, Solca F, Bauer MJ and Müllauer L (2021) Case Report: Afatinib Treatment in a Patient With NSCLC Harboring a Rare EGFR Exon 20 Mutation.

Front. Oncol. 10:593852. doi: 10.3389/fonc.2020.593852

\section{Case Report: Afatinib Treatment in a Patient With NSCLC Harboring a Rare EGFR Exon 20 Mutation}

\author{
Sabine Zöchbauer-Müller ${ }^{1,2 *}$, Bettina Kaserer ${ }^{3}$, Helmut Prosch ${ }^{4}$, Agnieszka Cseh ${ }^{5}$, \\ Flavio Solca ${ }^{6}$, Markus Johann Bauer ${ }^{6}$ and Leonhard Müllauer ${ }^{2,3}$ \\ ${ }^{1}$ Clinical Division of Oncology, Department of Medicine I, Medical University of Vienna, Vienna, Austria, ${ }^{2}$ Comprehensive \\ Cancer Center, Vienna, Austria, ${ }^{3}$ Institute of Pathology, Medical University of Vienna, Vienna, Austria, ${ }^{4}$ Department of \\ Biomedical Imaging and Image-Guided Therapy, Medical University of Vienna, Vienna, Austria, ${ }^{5}$ Boehringer Ingelheim \\ International GmbH, Ingelheim, Germany, ${ }^{6}$ Boehringer Ingelheim RCV GmbH \& Co. KG, Vienna, Austria
}

Unlike most other primary epidermal growth factor receptor (EGFR) mutations in nonsmall cell lung cancer (NSCLC), exon 20 insertions, comprising approximately 4\% to 10\% of all EGFR mutations, are generally considered to be resistant to EGFR tyrosine kinase inhibitors (TKIs). However, EGFR exon 20 insertions are structurally and pharmacologically heterogeneous, with variability in their position and size having implications for response to different EGFR TKIs. The second-generation ErbB family blocker, afatinib, is approved for the first-line treatment of EGFR mutation-positive NSCLC and has been shown to have a broad inhibitory profile against common and uncommon EGFR mutations. Here, we describe a patient with bilateral multifocal lung adenocarcinoma harboring a very rare EGFR exon 20 insertion (c.2317_2319dup3; p.H773dup), who has been receiving treatment with afatinib for 4.5 years. To our knowledge, this is the first report describing long-term benefit for a patient treated with afatinib with this rare exon 20 insertion. We are aware of two further cases with this rare EGFR mutation. One patient, also reported here, has early-stage lung adenocarcinoma and has not yet received systemic therapy for NSCLC. The other patient received afatinib in the context of a global compassionate use program and had progressive disease. Our findings may be of clinical relevance for patients carrying tumors with this rare mutation as epidemiological evidence suggests that P.H773dup may function as a driver mutation in NSCLC. Together with previous preclinical and clinical evidence for the activity of afatinib against certain EGFR exon 20 insertions, these findings warrant further investigation.

Keywords: afatinib, EGFR mutation, exon 20 insertion, H773dup, long-term response, NSCLC, uncommon mutation

\section{INTRODUCTION}

In non-small cell lung cancer (NSCLC), activating mutations in the epidermal growth factor receptor (EGFR) gene are reported in approximately $10 \%$ to $15 \%$ of Caucasian and $50 \%$ of Asian patients (1). EGFR mutation-positive tumors tend to be dependent on EGFR signaling for their growth and survival and, consequently, several EGFR-targeted therapeutics have been developed and approved. 
Two types of EGFR mutation, exon 19 deletions (Del19) and the exon 21 substitution L858R, represent approximately 45\% and $40 \%$ of all EGFR mutations, respectively (2). Del19 and L858R are therefore classed as common EGFR mutations and are the best characterized in terms of their association with response to EGFR TKIs (3). For these mutations, drugs approved by the United States (US) Food \& Drug Administration (FDA) for EGFR mutation-positive NSCLC comprise three generations of EGFR tyrosine kinase inhibitors (TKIs) - the first-generation reversible EGFR TKIs, erlotinib and gefitinib, the second-generation irreversible ErbB family blockers, afatinib and dacomitinib, and the third-generation irreversible, EGFR wild-type sparing TKI, osimertinib (4).

Many of the less common mutations are also sensitive to EGFR inhibitors. Afatinib, in particular, shows a broad preclinical activity across uncommon EGFR mutations (5), and has demonstrated clinical efficacy against uncommon mutations such as G719X, S768I, and L861Q (in exons 18, 20, and 21, respectively) (6). Based on these findings, the US indication for afatinib was extended to include S768I, L861Q and G719X mutations (7), while all activating EGFR mutations were already included in the labels in Europe (8).

Treatment options for the most prevalent uncommon mutations, i.e. EGFR exon 20 insertions ( $4-10 \%$ of all EGFR mutations), are not clear and represent an area of unmet need. A recent Phase 2 study, ZENITH20, assessed poziotinib, a covalent inhibitor of EGFR and human epidermal growth factor receptor 2 (HER2), in pretreated NSCLC patients with exon 20 insertions. Although the study did not meet its primary endpoint, there was some evidence of clinical activity, with an objective response rate (ORR) of $15 \%$, disease control rate of $69 \%$ and median progression-free survival (PFS) of 4.2 months. Treatment-naïve cohorts using alternative dosing regimens to improve tolerability are ongoing (9). Other investigational agents, including the EGFR/HER2 inhibitor, TAK-788 (10) and the bispecific EGFR/cMET antibody, JNJ-372 (11), are in clinical development. While, in principle, most EGFR exon 20 insertions could be considered as oncogenic driver mutations, since they promote interleukin 3- and EGF-independent growth of $\mathrm{Ba} / \mathrm{F} 3$ cells (5), structural and pharmacological differences between specific mutations mean that their sensitivity to targeted treatment differs depending on the inhibitor used and the mutational context. Some exon 20 insertions exist as compound mutations, which could also contribute to EGFRTKI resistance (5). With the exception of A763_Y764insFQEA (12), most EGFR exon 20 insertion mutations are resistant to first-generation EGFR TKIs, while the efficacy of second-/thirdgeneration TKIs against these mutations is less clear (13). Indeed, a post-hoc analysis of the LUX-Lung 2,3, and 6 trials suggested limited activity of afatinib treatment in patients with EGFR exon 20 insertions (6). In 23 such patients the ORR was $8.7 \%$ and median PFS was 2.7 months (6). In contrast, data from the afatinib uncommon EGFR mutations database indicate that afatinib has modest but apparent clinical activity. The response rate in 70 TKI-naïve patients with exon 20 insertions was $24 \%$ and median duration of response was 11.9 months
(14). In addition to the mutations known to be responsive to EGFR TKIs, a number of rare exon 20 insertions showed sensitivity to afatinib including A767delinsASVD (13) and A767_S768insSVA (15).

Previously published preclinical evidence shows that afatinib displays inhibitory activity against some EGFR exon 20 insertion mutations $(5,16-18)$. Using ectopically expressed EGFR-mutants in NIH-3T3 cell lines, we found that, consistent with previous findings, afatinib was at least 100 -fold more potent against G719S (Exon 18) and L861Q (Exon 21) mutations (Figure 1) compared to erlotinib. For most exon 20 mutations, EGFR phosphorylation was inhibited by afatinib at concentrations exceeding the clinically relevant $\mathrm{C}_{\max }$ of $100 \mathrm{nM}$ (except D770_N771insNPG), while erlotinib was ineffective in reducing constitutive phosphorylation in the exon 20 mutations tested at concentrations up to $10000 \mathrm{nM}$ (Figure 1). The observed biomarker modulation data is in-line with previously reported proliferation data in the $\mathrm{BA} / \mathrm{F} 3$ system $\left(\mathrm{IC}_{50}\right.$ of afatinib for Y764_V765insHH, A767_V769dupASV, and D770_N771insNPG were 134, 158, and $43 \mathrm{nM}$, respectively) $(10,16)$. Taken together, these data show differential sensitivity of EGFR exon 20 mutations to different EGFR-TKIs in preclinical and clinical contexts. Careful evaluation of the EGFR mutational context, including potency and therapeutic window, will be essential to select appropriate treatments for patients harboring tumors with EGFR exon 20 mutations.

Here, we report on a case of a patient with a very rare EGFR exon 20 insertion (c.2317_2319dup3; p.H773dup) who has been receiving treatment with afatinib for 4.5 years. In addition, we describe a patient in the early stages of lung cancer treatment, who underwent surgery at the same institution and had an identical p.H773dup mutation.

\section{CASE REPORT 1}

Patient 1 was a 62-year-old, female, ex-smoker (20 pack-years) with no major comorbidities. She was incidentally diagnosed in November 2014 with a $6.4 \mathrm{~cm}$ mass in the left upper lobe and multiple ground glass nodules in both lungs, the largest of which was located in the right middle lobe, with a maximum diameter of $1.8 \mathrm{~cm}$. Histopathology following resection of the left upper lobe confirmed lung adenocarcinoma (Grade 2; pT2b pN1 [2/17 lymph nodes positive]; V0 R0), with partially papillary and partially tubular morphology. Molecular pathology based on Sanger sequencing of EGFR exons 18 to 21 indicated no EGFR mutation, and the tumor was $A L K$ fluorescence in situ hybridization negative and ROS1 immunohistochemistrynegative (Figure 2).

The patient received 4 cycles of adjuvant chemotherapy with carboplatin and vinorelbine from January 2015 until April 2015, but a computed tomography (CT) scan in May 2015 showed disease progression. The scan revealed several pulmonary focal ground-glass opacities (GGOs) in both lungs, some of which had increased in size compared with preoperative CT findings (Figure 3). Biopsy of a GGO lesion of the right upper lobe in 


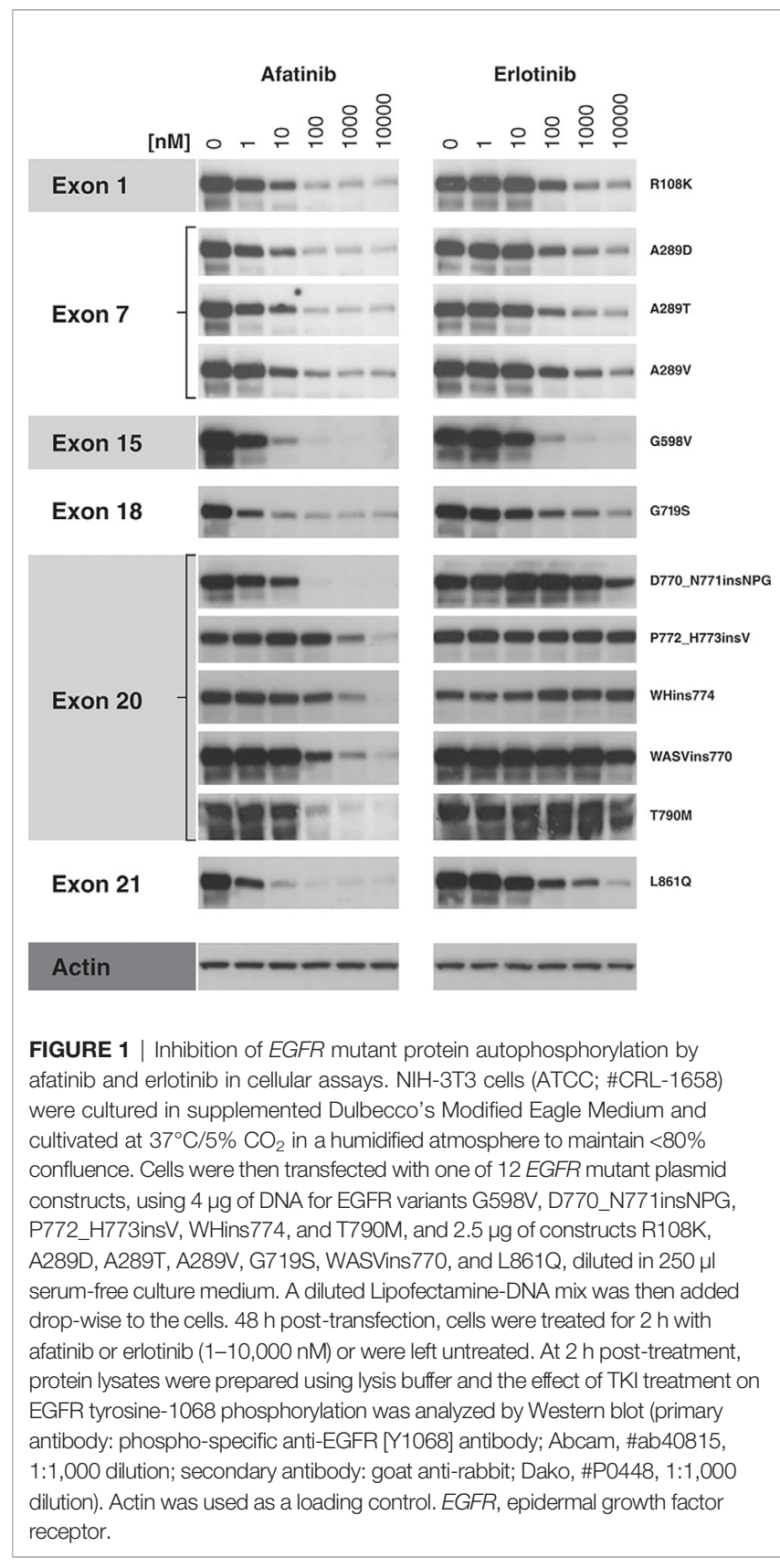

June 2015 indicated Grade 2 adenocarcinoma of the lung that was partially tubular and partially lepidic.

Further Sanger sequencing detected an EGFR exon 20 insertion mutation (NM_005228.3 [EGFR]: codon 2317_ 2319dupCAC; p.H773dup; Figures 4A, B). From July 2015, the patient received afatinib $40 \mathrm{mg} /$ day, which was reduced to $30 \mathrm{mg} /$ day in August 2015 and further reduced to $20 \mathrm{mg} /$ day in October 2015 due to diarrhea. The patient achieved stable disease until May 2016, followed by progression, which was treated with stereotactic irradiation to two lesions (40.5 Grays in 3 fractions to each lesion) in the left lower lobe and one lesion in the right upper lobe. She remained on treatment with afatinib (20 mg/day) and had stable disease again from July 2016 until July 2017 (Figure 3), when progression ensued and she received stereotactic irradiation to one lesion in the left lower lobe.

Two liquid biopsies were performed, in January and August 2017. No EGFR or any other mutation was detected in either sample, using next-generation sequencing (NGS) with a colon/ lung 22-gene (including EGFR) panel from Thermo Fisher Scientific. The sample from January was additionally analyzed with digital PCR for Del19 and T790M mutations, with none detected. In December 2018, a third liquid biopsy was performed, this time employing an NGS liquid biopsy lung circulating free DNA (11-gene) panel from Thermo Fisher Scientific. Again, no mutations were detected.

Ileocecal resection was performed in April 2018 because of ischemic necrosis of the cecum, and no carcinoma infiltration was detected in the resection specimen. Because of slightly enlarged retrocaval lymph nodes in a CT scan from December 2018, lymph node and lung biopsies were performed in January 2019. Endobronchial ultrasound-guided biopsy of the mediastinal lymph nodes revealed no carcinoma infiltration, and bronchoscopy with biopsy lower right lobe (B10) identified no carcinoma in the lung parenchyma. However, Escherichia coli and Klebsiella pneumonia were detected in the bronchoalveolar lavage, and the patient received antibiotic treatment with ciprofloxacin. A CT scan in February 2019 showed a decrease in size of the previously enlarged lymph nodes. Treatment with afatinib is ongoing as of October 2020, and the patient continues to have stable disease (Figure 3), with an Eastern Cooperative Oncology Group performance status of 0 .

\section{CASE REPORT 2}

Patient 2 was referred to our clinic for surgery in September 2019. The patient had previously undergone resection of breast carcinoma, and received radiotherapy and hormone ablation therapy. A lung tumor was detected with position emission tomography-CT scan, and resection of the left lower lobe with lymphadenectomy was performed in September 2019, due to suspicion of lung metastasis of the breast carcinoma. Histology revealed invasive adenocarcinoma, and NGS with the Oncomine Focus Assay (Thermo Fisher Scientific) identified an EGFR exon 20 insertion (NM_005228.3 [EGFR]: codon 2317_2319dupCAC; p.H773dup; Figures 4C, D) that was identical to the mutation identified in Patient 1.

After tumor resection, the patient was re-transferred to their previous hospital.

\section{DISCUSSION AND CONCLUDING REMARKS}

Exon 20 insertion mutations are the third most common type of EGFR mutation, after Del19 and L858R (3). Similar to Del19 and L858R, EGFR exon 20 insertions can result in sustained EGFR signaling and function as oncogenic drivers. However, 


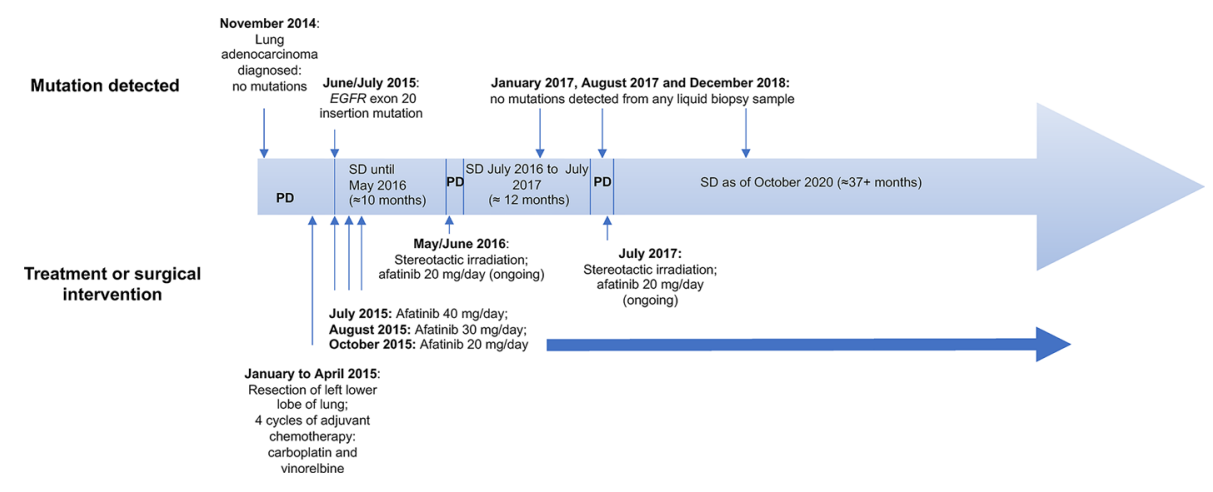

FIGURE 2 | Patient 1 case history and time line of key events. PD, progressive disease; SD, stable disease.

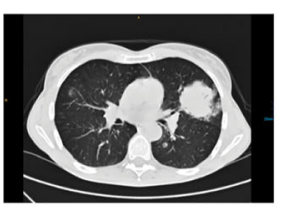

Initial CT, pre-surgery (November 2014)

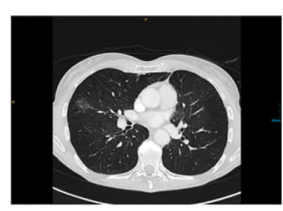

Pre-biopsy (May 2015)

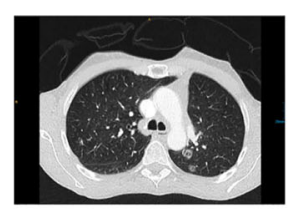

Disease progression (June 2016)

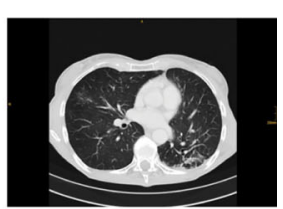

Stable disease (May 2019)

FIGURE 3 | Patient 1 clinical course, including treatment history and CT scans. CT, computed tomography.

despite their importance as potentially targetable mutations, the clinicopathologic characteristics and molecular spectrum of exon 20-mutant tumors have not been explored in most patient populations, and the biology underlying the heterogeneous responses of different genomic variants to targeted therapies is not well understood (3).

The first case presented herein describes a patient with NSCLC harboring an EGFR exon 20 insertion mutation who achieved durable stable disease with afatinib and remains on treatment after 4.5 years. To the best of our knowledge, this is the first report describing long-term benefit for a patient treated with afatinib with this rare exon 20 insertion. We are aware of two further cases with this same, rare EGFR mutation. One patient, also reported here, has early-stage lung adenocarcinoma and has not yet received systemic therapy for NSCLC. The other patient received afatinib in the context of a global compassionate use program and had progressive disease $(19,20)$. It is not uncommon for patients to have concurrent mutations alongside exon 20 insertions (21); therefore, we speculate that the difference in afatinib response could be owing to the presence of an additional mutation. For example, TP53 mutations are one of the most common concurrent mutations alongside exon 20 insertion (21) and is possibly associated with a lower likelihood of response to EGFR TKIs (22). Overall, these findings are particularly interesting and suggest that afatinib may provide a new therapeutic option for the particular type of mutation discussed here.
The exon 20 H773dup insertion, annotated as H773_ V774insH, has been reported previously, although such reports have not provided evidence for a potential driver role for this particular mutation $(3,23)$. To determine its prevalence in current databases, we searched for the occurrence of H773dup within the American Association for Cancer Research (AACR) Genomics Evidence Neoplasia Information Exchange (GENIE) database (The AACR Project GENIE Consortium, release 5.0), which comprises almost 60,000 samples across 81 major cancer types (24). We found 15 cases of H773dup in NSCLC, glioma, and endometrial cancer, showing prevalences of $0.12 \%, 0.06 \%$, and $0.12 \%$, respectively (Table 1A). In line with Qin et al. (21), we also find co-mutations in cancer-related genes like TP53 (4/15), PIK3CA (1/15), or PTEN (1/15) which might contribute to different responses upon treatment with TKIs. As a second source, we queried FoundationCore (version MI20190726), a proprietary database provided by Foundation Medicine. FoundationCore contains almost 300,000 clinical specimens and represents, to our knowledge, the biggest available database of its kind, allowing us to exhaustively describe the H773dup mutation landscape. In line with the GENIE results, we found H773dup mutations in NSCLC (adenocarcinoma and squamous cell carcinoma) and glioma (glioblastoma and anaplastic astrocytoma) at a prevalence of $0.1 \%$, as well as in other (niche) indications. Table 1B lists all instances of H773dup, highlighting its widespread occurrence. In addition to mutation information, FoundationCore provides 


\section{A}

EGFR exon 20

wild-type sequence in patient 1

B

EGFR exon 20

CAC duplication in patient 1
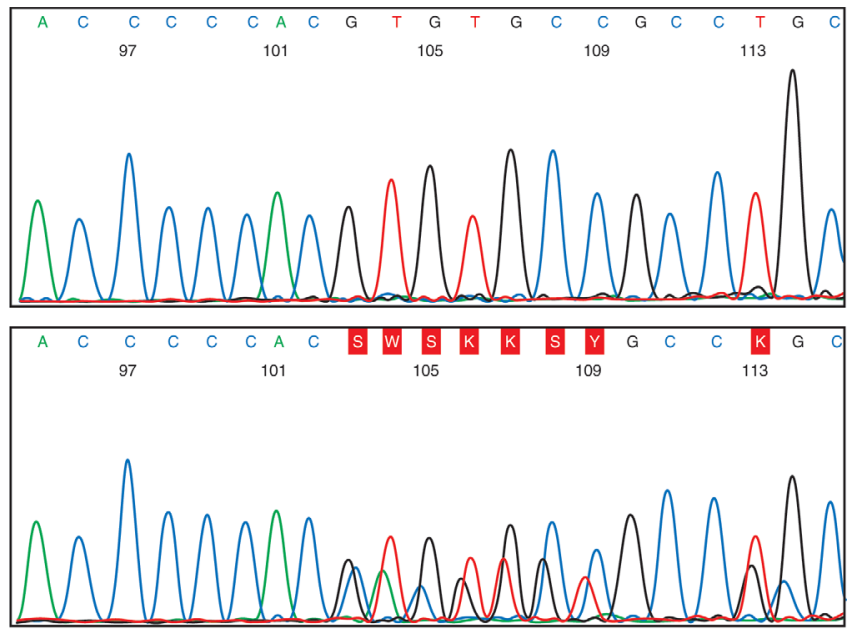

C

EGFR exon 20 wild-type sequence in patient 2

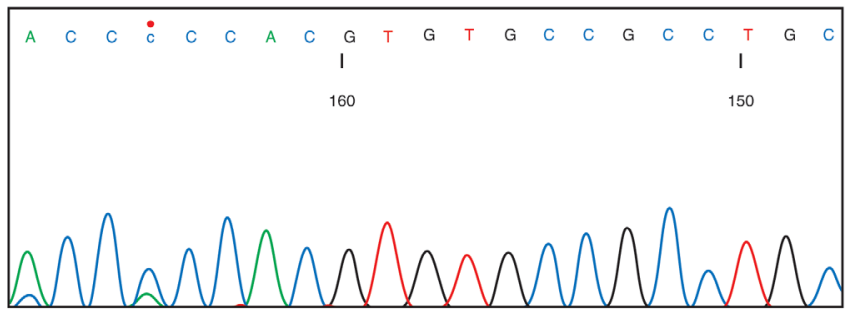

D

EGFR exon 20

CAC duplication in patient 2

FIGURE 4 | Insertion site of EGFR exon 20 insertion mutation. Sequencing electropherogram of EGFR exon 20 showing: (A) wild-type sequence of the adenocarcinoma from patient 1 resected in 2014; (B) duplication of a CAC base triplet in the adenocarcinoma from patient 1 biopsied in 2015 . EGFR, epidermal growth factor receptor; CAC, cytosine adenine cytosine; (C) wild-type sequence of the adenocarcinoma from patient 2 resected in 2019 ; (D) duplication of a CAC base triplet in the adenocarcinoma from patient 2 biopsied in 2019.

copy-number information for all samples. Interestingly, 33\% and $43 \%$ of lung adenocarcinomas of glioblastomas, respectively, show co-occurring EGFR amplifications, which are indicative of H773dup having a functional role. This corroborates an earlier claim that EGFR exon 20 insertions may function as driver mutations that are potentially susceptible to effective targeted therapy, based on exon 20 insertions being enriched in neversmokers and Asian patients (23).

The discrepancy in the results of EGFR mutation testing in 2014 and 2015 may at least partly reflect the fact that Patient 1 had a multifocal adenocarcinoma, as high rates of discordance in EGFR mutation status and subtype have been observed between GGO lesions from the same patient $(25,26)$. Alternatively, this could be attributable to emergence of a secondary EGFR mutation after chemotherapy. The clinical implications of such spatial and temporal heterogeneity are that the use of multiple biopsies on multiple lesions and repeated biopsies upon progression, together with the use of sensitive techniques such as NGS, may be needed to fully characterize the molecular pathology of multifocal adenocarcinomas before and after treatment.

Afatinib was generally well tolerated, and diarrhea adverse events (AEs) were managed effectively with dose reductions to $20 \mathrm{mg} /$ day, enabling Patient 1 to remain on afatinib treatment while still experiencing clinical benefit. This finding is consistent with previous reports that tolerability-guided dose reductions of afatinib are effective in mitigating drug-related AEs, without compromising efficacy (27).

EGFR exon 20 insertions are highly variable in position and size, with structural heterogeneity having implications for response to EGFR TKIs $(3,28)$. An in-silico modelling study predicted that insertions between codons 769 and 775 may be resistant to currently available EGFR TKIs, while insertions proximal to codon 769 are predicted to retain sensitivity (3). Additionally, it appears that different genomic variants confer heterogeneity in response to different EGFR-targeted therapies. Hirano et al. performed MTS 
TABLE 1 | Occurrence of H773dup mutations in the AACR Project GENIE and FoundationCore databases.

\begin{tabular}{|c|c|c|c|c|}
\hline \multicolumn{5}{|l|}{ A) AACR Project GENIE } \\
\hline Glioma & 3214 & 2 & 0.062 & $0.008-0.225$ \\
\hline Endometrial cancer & 1668 & 2 & 0.120 & $0.015-0.432$ \\
\hline Lung adenocarcinoma & 33096 & 24 & 0.1 & 33.33 \\
\hline Brain glioblastoma & 6162 & 7 & 0.1 & 42.86 \\
\hline Lung NSCLC (Nos) & 7197 & 5 & 0.1 & 20.00 \\
\hline Bladder urothelial (transitional cell) carcinoma & 3789 & 4 & 0.1 & 0 \\
\hline Kidney urothelial carcinoma & 603 & 2 & 0.3 & 100.00 \\
\hline Ovary serous carcinoma & 7216 & 1 & 0.01 & 100.00 \\
\hline Lung typical carcinoid & 72 & 1 & 1.4 & 0 \\
\hline Brain anaplastic astrocytoma & 887 & 1 & 0.1 & 0 \\
\hline Uterus endometrial adenocarcinoma & 1854 & 1 & 0.1 & 0 \\
\hline
\end{tabular}

AACR, American Association for Cancer Research; EGFR, epidermal growth factor receptor; GENIE, Genomics Evidence Neoplasia Information Exchange; NSCLC, non-small cell lung cancer; Nos, not otherwise specified.

assays using cells harboring four different types of EGFR exon 20 insertions. Afatinib potently inhibited the growth of cells harboring EGFR A763_Y764insFQEA ( $\mathrm{IC}_{50} 3 \mathrm{nM}$ vs $44 \mathrm{nM}$ for osimertinib), and afatinib and osimertinib showed similar efficacy against Y764_V765insHH (134 vs 237 nM), A767_V769dupASV (158 vs $333 \mathrm{nM}$ ), and 770_N771insNPG (43 vs $42 \mathrm{nM}$ ) (16). In contrast, erlotinib and rociletinib were relatively ineffective against these mutations (16). Our own preclinical findings also demonstrated heterogeneous responses, with exon 20 mutations at different amino acid positions showing a range of sensitivities to afatinib, while being largely insensitive to erlotinib (example in Figure 1). A retrospective analysis of Chinese patients, in which 85 unique EGFR exon 20 insertion variants were identified in 547 cases (of 24,468 patients screened) indicated heterogeneous response to EGFR TKIs in the clinic. PFS differed significantly among six representative EGFR exon 20 insertion variants $(\mathrm{p}=0.017)$ with p.A763_Y764insFQEA associated with better PFS than other insertions. Afatinib and osimertinib were associated with higher disease control rate than first-generation TKIs (21).

Emerging clinical evidence further supports the hypothesis that some exon 20 insertion mutations may be sensitive to afatinib. Among patients treated with afatinib as part of the global Named Patient Use (NPU) program, 100/723 patients with any EGFR mutations had uncommon mutations, including 20 patients with exon 20 insertions (20). The ORR among these patients was $35 \%$ (vs $23.4 \%$ in the overall NPU population). Cases have also been reported of patients with EGFR exon 20 insertions responding to afatinib. In one report, two patients with de novo EGFR exon 20 insertions (D770_N771insSVD and Ser768_Asp770dup) showed rapid clinical improvement after afatinib treatment, but disease progression quickly ensued in one patient (29). Although the authors concluded that responses to afatinib could be short lived in some patients (29), another case study of a patient with an A767_S768insSVA tandem duplication demonstrated a durable response to afatinib, with the patient surviving for over 3 years from the start of treatment (15). In a separate report, one patient with exon 20 insertion (initially H773_V774insH, D770_N771insG, V769_D770insASV, D770_N771insSVD) was treated with osimertinib, but mutation testing following progression suggested that the mutation site had changed to A767delinsASVD only. The patient subsequently received afatinib treatment, during which the primary tumor regressed and pleural effusion was significantly reduced, with a PFS of 7.4 months (13).

An alternative strategy that has been tested against tumors with EGFR exon 20 insertions is to combine EGFR TKIs with antiEGFR monoclonal antibody treatment. Preclinical evaluation of afatinib or osimertinib plus cetuximab demonstrated a mild but statistically significant additive antitumor effect of these combinations against several EGFR exon 20 insertion mutations in vitro. Afatinib plus cetuximab also significantly inhibited the growth of tumors harboring EGFR A767_V769dupASV and EGFR Y764_V765insHH, in vivo, while single-agent treatments did not (30). With regard to clinical data, among four patients with EGFR exon 20 insertions treated with afatinib plus cetuximab in the Netherlands, three patients had a partial response (PR), and the median PFS was 5.4 months (31).

Unlike EGFR exon 20 insertions, the spectrum of HER2 exon 20 mutations in NSCLC is narrower, with A775_G776insYVMA accounting for most cases (18). Nevertheless, as with EGFR exon 20 insertions, similar heterogeneity in responses of different HER2 exon 20 insertions to afatinib has been reported. One study 
investigating specific HER2 exon 20 insertions in a Chinese cohort found that patients with tumors harboring G778_P780dup achieved numerically longer median PFS ( 10 vs 3.3 months, $\mathrm{p}=0.32$ ) and overall survival ( 19.7 vs 7 months, $\mathrm{p}=0.16$ ) with afatinib versus nonG778 patients, which is consistent with in vitro results suggesting that Glycine778 may facilitate inhibitor binding to HER2 (32). Among patients who received afatinib in a global NPU program, 12 patients with information available on the type of HER2 mutation had an exon 20 mutation, among whom 10 patients (83\%) had A775_G776insYVMA (33). Four of these patients remained on afatinib for more than 1 year, and this subgroup demonstrated a median time to treatment failure of 9.6 months, compared with just 1.9 months in the other two patients, both with M774 duplications.

Preclinical studies also suggest that irreversible EGFR TKIs such as afatinib and dacomitinib are active against HER2 exon 20 insertions, but at $\sim 100$-fold higher concentrations than are necessary to inhibit Del19 or L858R models $(34,35)$. Consequently, Costa et al. tested intermittent pulsatile doses of afatinib in preclinical models of NSCLC with HER2 exon 20 insertions, with the aim of achieving intermittent plasma concentrations that would exceed the threshold for efficacy, while improving tolerability versus daily dosing. Pulse afatinib induced anti-tumor activity in these models, and evidence of clinical activity (one PR and one stable disease) was observed among three patients with advanced HER2 exon 20 insertionmutated NSCLC treated with off-label pulse afatinib (36). Overall, there is accumulating evidence that afatinib may provide a viable therapeutic option for patients with at least some types of EGFR and HER2 exon 20 insertion, with different approaches having been evaluated in this difficult-to-treat population.

Regarding other treatment options, a Phase 2 trial of poziotinib did not meet its primary endpoint, however the trial is ongoing in other cohorts: treatment-naive NSCLC patients with exon 20 insertions and alternative dosing regimens to improve tolerability (9). However, despite preliminary activity, a request for breakthrough therapy designation for poziotinib for the treatment of EGFR exon 20 insertion mutation-positive NSCLC was rejected by the FDA. Currently, there are no treatments approved in this particular indication, although other TKIs designed to target exon 20 insertions, such as TAK-788, which has received breakthrough therapy designation from the FDA, are in early clinical development (37).

At the time that afatinib treatment was initiated in our patient, there was a lack of investigational treatments for patients with EGFR exon 20 insertions, and chemotherapy was and still is the standard treatment choice for these patients. In our case, the patient relapsed shortly after adjuvant chemotherapy, and afatinib treatment was chosen after detection of an exon 20 insertion mutation due to its broad inhibitory profile against uncommon EGFR mutations.

In conclusion, our report describes two rare cases of patients with H773dup, one of whom was treated with afatinib for 4.5 years (still on treatment at the time of this report). Our findings are in line with epidemiological evidence that this very rare $(\sim 0.1 \%$ prevalence in NSCLC), albeit widespread (across tumor types), mutation has a functional role as a driver mutation in NSCLC and can be treated with appropriate EGFR-targeted therapy. Finally, the long time on treatment and durable stable disease observed in Patient 1 is testament to afatinib's manageable safety profile, and suggests that afatinib may be a viable therapeutic option for patients with tumors harboring this exon 20 insertion mutation, particularly those for whom chemotherapy is unsuccessful. Together with previous preclinical and clinical evidence supporting afatinib's activity against certain EGFR exon 20 insertions, these findings warrant further investigation.

\section{DATA AVAILABILITY STATEMENT}

The original contributions presented in the study are included in the article/supplementary material, further inquiries can be directed to the corresponding author/s.

\section{ETHICS STATEMENT}

Ethical review and approval was not required for the study on human participants in accordance with the local legislation and institutional requirements. The patients/participants provided their written informed consent to participate in this study. Written informed consent was obtained from both participants for the publication of this case report and any potentially identifying information/images.

\section{AUTHOR CONTRIBUTIONS}

SZM, BK, MB, and LM collected and assembled the data, analyzed and interpreted the data, and drafted the manuscript. HP analyzed and interpreted the data, and drafted the manuscript. AC conceived and designed the study, analyzed and interpreted the data, and drafted the manuscript. FS conceived and designed the study, collected, assembled, analyzed and interpreted the data, and drafted the manuscript. All authors provided final approval of the manuscript and agreed to be accountable for all aspects of the work, which includes ensuring that questions related to the accuracy or integrity of any part of the work are appropriately investigated and resolved. All authors contributed to the article and approved the submitted version.

\section{FUNDING}

The authors would like to acknowledge the American Association for Cancer Research and its financial and material support in the development of the AACR Project GENIE registry, as well as members of the consortium for their commitment to data sharing. Interpretations are the responsibility of the study authors. Medical writing assistance, supported financially by Boehringer Ingelheim, was provided by Fiona Scott, $\mathrm{PhD}$, of GeoMed, an Ashfield company, part of UDG Healthcare plc, during the preparation of this article. 


\section{REFERENCES}

1. Chan BA, Hughes BG. Targeted therapy for non-small cell lung cancer: current standards and the promise of the future. Transl Lung Cancer Res (2015) 4:36-54. doi: 10.3978/j.issn.2218-6751.2014.05.01

2. Kim EY, Cho EN, Park HS, Hong JY, Lim S, Youn JP, et al. Compound EGFR mutation is frequently detected with co-mutations of actionable genes and associated with poor clinical outcome in lung adenocarcinoma. Cancer Biol Ther (2016) 17:237-45. doi: 10.1080/15384047.2016.1139235

3. Arcila ME, Nafa K, Chaft JE, Rekhtman N, Lau C, Reva BA, et al. EGFR exon 20 insertion mutations in lung adenocarcinomas: prevalence, molecular heterogeneity, and clinicopathologic characteristics. Mol Cancer Ther (2013) 12:220-9. doi: 10.1158/1535-7163.Mct-12-0620

4. Girard N. Optimizing outcomes in EGFR mutation-positive NSCLC: which tyrosine kinase inhibitor and when? Future Oncol (2018) 14:1117-32. doi: 10.2217/fon-2017-0636

5. Kohsaka S, Nagano M, Ueno T, Suehara Y, Hayashi T, Shimada N, et al. A method of high-throughput functional evaluation of EGFR gene variants of unknown significance in cancer. Sci Transl Med (2017) 9:eaan6566. doi: 10.1126/scitranslmed.aan6566

6. Yang JC, Sequist LV, Geater SL, Tsai CM, Mok TS, Schuler M, et al. Clinical activity of afatinib in patients with advanced non-small-cell lung cancer harbouring uncommon EGFR mutations: a combined post-hoc analysis of LUX-Lung 2, LUX-Lung 3, and LUX-Lung 6. Lancet Oncol (2015) 16:830-8. doi: 10.1016/s1470-2045(15)00026-1

7. FDA. Gilotrif ${ }^{\circledR}$ (afatinib) Highlights of Prescribing Information (2018). Available at: https://docs.boehringer-ingelheim.com/Prescribing\% 20Information/PIs/Gilotrif/Gilotrif.pdf (Accessed July 4, 2019).

8. EMA. Giotrif (EPAR) Summary of Product Characteristics (2018). Available at: https://www.ema.europa.eu/en/documents/product-information/giotrifepar-product-information_en.pdf (Accessed July 4, 2019).

9. Le X, Goldman JW, Clarke JM, Tchekmedyian N, Piotrowska Z, Chu D, et al. Poziotinib shows activity and durability of responses in subgroups of previously treated EGFR exon 20 NSCLC patients. J Clin Oncol (2020) 38: Abstract9514. doi: 10.1200/JCO.2020.38.15_suppl.9514

10. Janne PA, Neal JW, Camidge DR, Spira AI, Piotrowska Z, Horn L, et al. Antitumor activity of TAK-788 in NSCLC with EGFR exon 20 insertions. J Clin Oncol (2019) 37(Suppl 15):Abstract9007. doi: 10.1200/ JCO.2019.37.15_suppl.9007

11. Haura EB, Cho BC, Lee JS, Han J-Y, Lee KH, Sanborn RE, et al. JNJ-61186372 (JNJ-372), an EGFR-cMet bispecific antibody, in EGFR-driven advanced nonsmall cell lung cancer (NSCLC). J Clin Oncol (2019) 37(Suppl 15): Abstract9009. doi: 10.1200/JCO.2019.37.15_suppl.9009

12. Yasuda H, Park E, Yun C-H, Sng NJ, Lucena-Araujo AR, Yeo W-L, et al. Structural, biochemical, and clinical characterization of epidermal growth factor receptor (EGFR) exon 20 insertion mutations in lung cancer. $J$ Sci Transl Med (2013) 5:216ra177. doi: 10.1126/scitranslmed.3007205

13. Cai Y, Wang X, Guo Y, Sun C, Xu Y, Qiu S, et al. Successful treatment of a lung adenocarcinoma patient with a novel EGFR exon 20-ins mutation with afatinib: a case report. Medicine (Baltimore) (2019) 98:e13890. doi: 10.1097/ md.0000000000013890

14. Yang JC, Schuler M, Popat S, Miura S, Heeke S, Park K, et al. Afatinib for the treatment of NSCLC harboring uncommon EGFR mutations: a database of 693 cases. J Thorac Oncol (2020) 15:803-15. doi: 10.1016/j.jtho.2019.12.126

15. Chan RT. Afatinib for an EGFR exon 20 insertion mutation: a case report of progressive stage IV metastatic lung adenocarcinoma with 54 months' survival. Asia Pac J Clin Oncol (2018) 14(Suppl 1):7-9. doi: 10.1111/ajco.12853

16. Hirano T, Yasuda H, Tani T, Hamamoto J, Oashi A, Ishioka K, et al. In vitro modeling to determine mutation specificity of EGFR tyrosine kinase inhibitors against clinically relevant EGFR mutants in non-small-cell lung cancer. Oncotarget (2015) 6:38789-803. doi: 10.18632/oncotarget.5887

17. Kobayashi Y, Mitsudomi T. Not all epidermal growth factor receptor mutations in lung cancer are created equal: Perspectives for individualized treatment strategy. Cancer Sci (2016) 107:1179-86. doi: 10.1111/cas.12996

18. Kosaka T, Tanizaki J, Paranal RM, Endoh H, Lydon C, Capelletti M, et al. Response heterogeneity of EGFR and HER2 exon 20 insertions to covalent EGFR and HER2 inhibitors. J Cancer Res (2017) 77:2712-21. doi: 10.1158/ 0008-5472.CAN-16-3404
19. Uncommon EGFR Mutations. Available at: https://www.uncommonegfr mutations.com/Results/Search\# (Accessed April 30, 2020).

20. Cappuzzo F, Soo R, Hochmair M, Schuler M, Lam KC, Stehle G, et al. Global named patient use program of afatinib in advanced non-small-cell lung carcinoma patients who progressed following prior therapies. Future Oncol (2018) 14:1477-86. doi: 10.2217/fon-2017-0666

21. Qin Y, Jian H, Tong X, Wu X, Wang F, Shao YW, et al. Variability of EGFR exon 20 insertions in 24468 Chinese lung cancer patients and their divergent responses to EGFR inhibitors. Mol Oncol (2020) 14:1695-704. doi: 10.1002/ 1878-0261.12710

22. Canale M, Petracci E, Delmonte A, Bronte G, Chiadini E, Ludovini V, et al. Concomitant TP53 mutation confers worse prognosis in EGFR-mutated nonsmall cell lung cancer patients treated with TKIs. J Clin Med (2020) 9:1047. doi: $10.3390 / \mathrm{jcm} 9041047$

23. Oxnard GR, Lo PC, Nishino M, Dahlberg SE, Lindeman NI, Butaney M, et al. Natural history and molecular characteristics of lung cancers harboring EGFR exon 20 insertions. J Thorac Oncol (2013) 8:179-84. doi: 10.1097/ JTO.0b013e3182779d18

24. The AACR Project GENIE Consortium. AACR Project GENIE: powering precision medicine through an international consortium. J Cancer Discov (2017) 7:818-31. doi: 10.1158/2159-8290.CD-17-0151

25. Liu M, He W-X, Song N, Yang Y, Zhang P, Jiang G-N. Discrepancy of epidermal growth factor receptor mutation in lung adenocarcinoma presenting as multiple ground-glass opacities. Eur J CardioThorac Surg (2016) 50:909-13. doi: 10.1093/ejcts/ezw113

26. Tanvetyanon T, Boyle TA. Clinical implications of genetic heterogeneity in multifocal pulmonary adenocarcinomas. J Thorac Dis (2016) 8:E1734-8. doi: 10.21037/jtd.2016.12.06

27. Yang JC-H, Sequist LV, Zhou C, Schuler M, Geater SL, Mok T, et al. Effect of dose adjustment on the safety and efficacy of afatinib for EGFR mutationpositive lung adenocarcinoma: post hoc analyses of the randomized LUXLung 3 and 6 trials. Ann Oncol (2016) 27:2103-10. doi: 10.1093/annonc/ mdw322

28. O'Kane GM, Bradbury PA, Feld R, Leighl NB, Liu G, Pisters KM, et al. Uncommon EGFR mutations in advanced non-small cell lung cancer. Lung Cancer (2017) 109:137-44. doi: 10.1016/j.lungcan.2017.04.016

29. Costa M, Lewgoy J. P3.13-13 Afatinib in lung adenocarcinoma harboring de novo EGFR exon 20 insertions. J Thorac Oncol (2018) 13:S981. doi: 10.1016/ j.jtho.2018.08.1853

30. Hasegawa H, Yasuda H, Hamamoto J, Masuzawa K, Tani T, Nukaga S, et al. Efficacy of afatinib or osimertinib plus cetuximab combination therapy for non-small-cell lung cancer with EGFR exon 20 insertion mutations. Lung Cancer (2019) 127:146-52. doi: 10.1016/j.lungcan.2018.11.039

31. van Veggel B, de Langen AJ, Hashemi SMS, Monkhorst K, Heideman DAM, Thunnissen E, et al. Afatinib and cetuximab in four patients with EGFR exon 20 insertion-positive advanced NSCLC. J Thorac Oncol (2018) 13:1222-6. doi: $10.1016 /$ j.jtho.2018.04.012

32. Liu Z, Wu L, Cao J, Yang Z, Zhou C, Cao L, et al. Clinical characterization of ERBB2 exon 20 insertions and heterogeneity of outcomes responding to afatinib in Chinese lung cancer patients. Onco Targets Ther (2018) 11:732331. doi: $10.2147 /$ ott.S173391

33. Peters S, Curioni-Fontecedro A, Nechushtan H, Shih J-Y, Liao W-Y, Gautschi O, et al. Activity of afatinib in heavily pretreated patients with ERBB2 mutationpositive advanced NSCLC: findings from a global named patient use program. J Thorac Oncol (2018) 13:1897-905. doi: 10.1016/j.jtho.2018.07.093

34. Engelman JA, Zejnullahu K, Gale CM, Lifshits E, Gonzales AJ, Shimamura T, et al. PF00299804, an irreversible pan-ERBB inhibitor, is effective in lung cancer models with EGFR and ERBB2 mutations that are resistant to gefitinib. Cancer Res (2007) 67:11924-32. doi: 10.1158/0008-5472.Can-07-1885

35. Li D, Ambrogio L, Shimamura T, Kubo S, Takahashi M, Chirieac LR, et al. BIBW2992, an irreversible EGFR/HER2 inhibitor highly effective in preclinical lung cancer models. Oncogene (2008) 27:4702-11. doi: 10.1038/ onc.2008.109

36. Costa DB, Jorge SE, Moran JP, Freed JA, Zerillo JA, Huberman MS, et al. Pulse afatinib for ERBB2 exon 20 insertion-mutated lung adenocarcinomas. J Thorac Oncol (2016) 11:918-23. doi: 10.1016/j.jtho.2016.02.016

37. Neal J, Doebele R, Riely G, Spira A, Horn L, Piotrowska Z, et al. P1.13-44 Safety, PK, and preliminary antitumor activity of the oral EGFR/HER2 exon 
20 inhibitor TAK-788 in NSCLC. J Thorac Oncol (2018) 13:S599. doi: 10.1016/ j.jtho.2018.08.901

Conflict of Interest: SZM reports advisory council or committee relationship with Boehringer Ingelheim, Roche, MSD, BMS, Takeda, and AstraZeneca, honoraria from Bayer and Pfizer, and grants or funds from MSD. HP reports advisory council or committee relationship with Boehringer Ingelheim, Roche, MSD, and honoraria from Boehringer Ingelheim, Roche, MSD, BMS, and AstraZeneca. AC, FS, and MB report employment with Boehringer Ingelheim. LM reports consulting fees (advisory boards) from Boehringer Ingelheim.

The authors declare that this case report received funding for medical writing assistance from Boehringer Ingelheim. The funder was involved in the conception and design, analysis and interpretation of data, knowledge generation from molecular data, the writing and reviewing of this article, the final approval, and the decision to submit it for publication.

The remaining authors declare that the research was conducted in the absence of any commercial or financial relationships that could be construed as a potential conflict of interest.

Copyright (ㅇ 2021 Zöchbauer-Müller, Kaserer, Prosch, Cseh, Solca, Bauer and Müllauer. This is an open-access article distributed under the terms of the Creative Commons Attribution License (CC BY). The use, distribution or reproduction in other forums is permitted, provided the original author(s) and the copyright owner(s) are credited and that the original publication in this journal is cited, in accordance with accepted academic practice. No use, distribution or reproduction is permitted which does not comply with these terms. 